\title{
Checklist of the genera Conocybe and Pholiotina (Agaricales, Agaricomycetes) in Estonia
}

\author{
Kuulo Kalamees $^{1,2}$, Anton Hausknecht ${ }^{3}$ \& Jukka Vauras ${ }^{4}$ \\ ${ }^{1}$ Institute of Ecology and Earth Sciences, University of Tartu, 14A Ravila St., 50411 Tartu, Estonia. \\ E-mail: kuulo.kalamees@ut.ee \\ ${ }^{2}$ Institute of Agricultural and Environmental Sciences, Estonian University of Life Sciences, \\ 5D Kreutzwaldi St., 51014 Tartu, Estonia. E-mail: kuulo.kalamees@emu.ee \\ ${ }^{3}$ Fakultätszentrum für Biodiversität der Universität Wien, Rennweg 14, A-1030 Wien, Austria. \\ E-mail: ahausknecht.oemg@aon.at \\ ${ }^{4}$ Biological Collections of Åbo Akademi University, Herbarium, FI-20014 University of Turku, Finland. \\ E-mail: jukka.vauras@utu.fi
}

\begin{abstract}
Conocybe and Pholiotina (Agaricales, Agaricomycetes) have been recorded in Estonia. A checklist of these taxa with notes on ecology, phenology and distribution is presented.
\end{abstract}

\section{INTRODUCTION}

A monograph of the agaricoid genera Conocybe Fayod and Pholiotina Fayod has been published recently (Hausknecht, 2009), as well as surveys of Conocybe from Finland (Hausknecht et al., 2005) and Bolbitius, Conocybe and Pholiotina from Norway (Hausknecht et al., 2011).

The present checklist contains 28 species and varieties of Conocybe and 10 of Pholiotina recorded in Estonia. All the taxa included correspond to the conceptions of taxa established by Hausknecht \& Vesterholt (2008a-b), Hausknecht (2009) and have been proved by relevant exsiccates in the fungaria TAAM (Institute of Agricultural and Environmental Sciences, Estonian University of Life Sciences), TUR, TUR-A (University of Turku, Finland) and H (Helsinki University, Finland). Specimens in fungaria have been collected by A. Bresinsky (Regensburg, Germany); A. Jakobson, K. Kalamees, H. Kelder, A. Kollom, V. Liiv, I. Lumiste, L. Pihlik, G. Shtshukin, M. Vaasma, S. Veldre (Estonia); P. Höijer, I. Kytövuori, J. Vauras (Finland) but identified and/or revised principally by A. Hausknecht (cf Hausknecht, 2005, 2009).

The checklist also provides information on the ecology, phenology and occurrence of these taxa in Estonia. The following data are presented on each taxon: (1) the Latin name with a reference to the initial source; (2) most important synonyms; (3) references to the most important and representative pictures (iconography) in the mycological literature used in identifying Estonian species; (4) data on the ecology, phenology and distribution; (5) references to the specimens available in fungal collections of Estonia and/ or Finland, using the internationally accepted abbreviations; (6) comments.

Paal (1997) classification of forest types were used in order to characterize the habitats, if possible. The frequency of the occurrence of taxa is estimated as follows: very rare $-1-2$, rare $-3-5$, rather rare $-6-10$ localities. The frequency of the occurrence of the taxa has been presented in low frequency classes since the species of the genera Conocybe and Pholiotina have been collected and identified on the basis of occasional finds, although most of them are represented more widely in Estonia.

Abbreviations of iconography are following:

BK: Breitenbach \& Kränzlin, 1995

CD: Courtecuisse \& Duhem, 1994, 2000

Cetto76: Cetto, 1976

Cetto87: Cetto, 1987

Cetto89: Cetto, 1989

Cetto93: Cetto, 1993

D: Dähncke, 2001-2004

H: Hausknecht, 2009

L: Ludwig, 2007

Phil: Phillips, 1981

RH: Ryman \& Holmåsen, 1984 


\section{LIST OF SPECIES AND VARIETIES}

\section{CONOCYBE Fayod, Ann. Sci. Nat. Sér. VII, 9: 357. 1889}

Conocybe AlbIPes (G.H. Otth) Hauskn., Österr. Z. Pilzk. 7: 102. 1998, var. ALBIPES

C. lactea (J.E. Lange) Métrod, Bull. Soc. Mycol. France 56: 46. 1940.

Icon.: BK 381, as C. lactea; Cetto87 1750, as C. lactea; H 31 a-e, h, pp.818-820; L 92.2; Phil p.155, as C. lactea; RH p.435, as C. lactea.

Ecol. \& Distr.:In oligo-mesotrophic boreal forest, September, very rare; one locality in Estonia: Põlva Co., Veriora Comm., Nohipalo, Meenikunno Landscape Reserve, near Valgjärv, in poplar-pine-spruce mixed forest, 13 Sep 2000, K. Kalamees.

Voucher specimens studied: TAAM 175647 (det. K. Kalamees, as C. lactea; det. A.

Hausknecht, as C. albipes); rev. K. Kalamees, as C. albipes var. albipes.

Conocybe ambigua Watling, Notes Roy. Bot. Gard. Edinburgh 38: 331. 1980

Icon.: BK 375; H 8 a-c, pp. 741-742.

Ecol. \& Distr.: In eutrophic boreo-nemoral forests, October, very rare; one locality (two records) in Estonia: Jõgeva Co., Saare Comm., Roela, Vooremaa Forest Ecology Station, in spruce forest of Hepatica site type, $3 \& 13$ Oct 1972, K. Kalamees \& A. Kollom.

Voucher specimens studied: TAAM 081876, 114231.

Conocybe Apala (Fr.: Fr.) Arnolds, Persoonia 18: 225. 2003

C. elegans Watling, Nordic. J. Bot. 3: 263. 1983. Icon.: Cetto89 2219, as C. bulbifera (Kauffman) Kühner; H 29 a-c, pp. 791-793; L 92.23, as C. elegans.

Ecol. \& Distr.: In eutrophic boreo-nemoral coniferous forests, especially in spruce forests of Aegopodium site type, also in eutrophic paludifying grasslands, on roadsides and in yards, in June, August and September, rare.

Voucher specimens studied: TAAM 076839a (det. T. Leisner, as C. lactea), 081027, 142705, 171748; TUR-A 139463 (det. A. Hausknecht, as C. elegans); H 7022264 (det. I. Kytövuori) (Hausknecht, 2005).

CONOCYBE BISPORA (Singer) Hauskn., Österr. Z. Pilzk. 7: 114. 1998

Icon.: H 8 d, e, pp. 782-783; L 92.15.
Ecol. \& Distr.: In grasslands, September and October, very rare; two localities in Estonia: Lääne Co., Matsalu National Park, Sipelga Islet, in epilittoral grassland, 8 Sep 1980, K. Kalamees (det. K. Kalamees, as C. lactea); Võru Co., Võru town, NE side of Tamula Lake, in meadow close to the beach, on sandy soil, 2 Oct 1997, J. Vauras 12989. (Hausknecht 2009: 382).

Voucher specimens studied: TAAM 121560; TUR-A 139448.

CONOCYBE BRACHYPOdII (Velen.) Hauskn. \& Svrček, Czech Mycol. 51: 43. 1999

Icon.: H 1 e, 3 a-c, pp.702-703; L 92.36. and 92.38, as C. microspora.

Ecol. \& Distr.: In lawn in pine forest, September, very rare; one locality in Estonia: Hiiu Co., Emmaste Comm., church yard with grass-herb lawn in open forest with Pinus sylvestris, on calcareous soil, 15 Sep 2001, J. Vauras 17877. Voucher specimens studied: TUR 155680.

CONOCYBE BRUNNEIDISCA (Murrill) Hauskn. in Hausknecht \& Contu, Österr. Z. Pilzk. 16: 159. 2007

C. lenticulospora Watling, Notes Roy. Bot. Gard. Edinburgh 38: 351. 1980.

Icon.: H 28 b, c, pp. 816-818; L 92.17, as C. lenticulospora.

Ecol. \& Distr.: In oligo-mesotrophic boreal and eutrophic alvar coniferous forests, in gardens, on soil or dung, June, July and November, rare. Voucher specimens studied: TAAM 080652, 095605, 144772, 146903 (all det. A. Hausknecht, as C. lenticulospora); all rev. K. Kalamees, as C. brunneidisca.

CONOCYBE Dumetorum (Velen.) Svrček var. laricina (Kühner) Hauskn., Czech Mycol. 51: 51. 1999 Icon.: H $11 \mathrm{c}, \mathrm{d}, \mathrm{pp} .834-835$; L 92.32.

Ecol. \& Distr.: In lawn, September, very rare; one locality in Estonia: Hiiu Co., Käina Comm., Kassari, under large Quercus robur near the museum, in lawn, 14 Sep 2001, J. Vauras 17839F. Voucher specimens studied: TUR 156895.

Conocybe echinata (Velen.) Singer, Fieldiana, Botany n.s. 21: 103. 1989

Icon.: Cetto89 2225, as C. sordida; CD 1327, as C. sordida; H 13 a-c, pp. 714-715: L. 92.34.C. Ecol. \& Distr.: In eutrophic boreo-nemoral and drained peatland forests, along grassy path and bush margins, September and October, rare. Voucher specimens studied: TUR-A 139459, 139461, 182184, 182185. 
Conocybe fuscimarginata (Murrill) Singer, Beih. Nova Hedwigia 29: 210. 1969

Icon.: BK 379; H. 22 d-f, pp. 800-801; L 92.22.AE.

Ecol. \& Distr.: Eutrophic boreo-nemoral and alvar coniferous forests, in grasslands, on lawns, on dung, rotting wood and soil, August and September, rare.

Voucher specimens studied: TAAM 121447 , 143805, 144321, 178631 ; TUR 155687.

Conocybe hornana Singer \& Hauskn., Beitr. Kenntn. Pilze Mitteleur. 5: 87. 1989, var. HORNANA Icon.: Cetto93 2656, as C. hornana; H 34 a-e, pp. 840-842; L 92.19, as C. hornana.

Ecol. \& Distr.: In orchard, June, very rare; one locality in Estonia: Tartu Co., Tartu town, 9 Palsami St., in orchard, on compost, 5 June 1996, K. Kalamees (Hausknecht 2009: 523).

Voucher specimens studied: TAAM 146904 (det. A. Hausknecht, as C. hornana); rev. K. Kalamees, as C. hornana var. hornana.

Conocybe Juniana (Velen.) Hauskn. \& Svrček in Hausknecht, Österr. Z. Pilzk. 8: 46. 1999, var. JUNIANA

C. magnicapitata P.D. Orton, Trans. Brit. Mycol. Soc. 43: 193. 1960.

Icon.: BK 382, as C. magnicapitata; D p.581, as C. juniana; H 13 d, e, pp. 718-719; L 92.29, as C. juniana, 92.34.A, as C. echinata, 92.41.A, as $C$. tenera.

Ecol. \& Distr.: In eutrophic boreo-nemoral forests, July and October, very rare; one locality (two records) in Estonia: Jõgeva Co., Saare Comm., Roela, Vooremaa Forest Ecology Station, in spruce forests of Hepatica site type, 2 Oct 1972, K. Kalamees \& A. Kollom.

Voucher specimens studied: TAAM 114080, 114230 (both det. A. Hausknecht, as C. juniana); both rev. K. Kalamees, as C. juniana var. juniana.

Conocybe Juniana (Velen.) Hauskn. \& Svrček var. SUBSEJUNCTA Hauskn., Österr. Z. Pilzk. 8: 52. 1999

Icon.: H 13 g, pp. 720-721; L 92.34.D, as C echinata.

Ecol. \& Distr.: In eutrophic boreo-nemoral forests, July and October, very rare; one locality (two records) in Estonia: Jõgeva Co., Saare Comm., Roela, Vooremaa Forest Ecology Station, in spruce forests of Hepatica site type, 10 July 1972, K. Kalamees, 3 Oct 1972, K. Kalamees \& A. Kollom.
Voucher specimens studied: TAAM 079677, 114278.

CONOCYBE MACROCEPHALA Kühner \& Watling in Watling, Notes Roy. Bot. Gard. Edinburgh 38: 335. 1980

C. abruptibulbosa Watling, Notes Roy. Bot. Gard. Edinburgh 38: 335. 1980.

Icon.: BK 374, as C. abruptibulbosa; Cetto89 2217; Cetto93 2653, as C. abruptibulbosa; H 1 a-c, pp. 673-674; L 92.30.A-D.

Ecol. \& Distr.: In wooded meadows, eutrophic boreo-nemoral forests, eutrophic alvar shrublands, in lawns, on soil, September, rare.

Voucher specimens studied: TAAM 121525, 141797; TUR 110427, 155760 (J. Vauras 17829F).

CONOcybe MESOSPORA Kühner \& Watling, Notes Roy. Bot. Gard. Edinburgh 38: 336. 1980

Icon.: H. 6 a-f, pp. 705-709; L 92.37.

Ecol. \& Distr.: In eutrophic boreo-nemoral forest, in leaf litter, September, very rare, one locality in Estonia: Saare Co., Kaarma Comm., Abruka Nature Reserve, Abruka Island, in broad-leaved forest of Aegopodium site type, 14 Sep 1993, I. Kytövuori 93-1230.

Voucher specimens studied: H 7022241.

Conocybe Ochrostriata Hauskn., Österr. Z. Pilzk. 14: 246. 2005, var. OCHROSTRIATA

C. sienophylla (Berk. \& Broome) Singer ss. auct. non ss. orig.; C. ochracea (Kühner) Singer, Mycologia 51: 395. 1959 (nom. inval.).

Icon.: BK 391, as C. sienophylla; Cetto93 2651, as C. sienophylla; H 26 b, d, f, p.765, as C. sienophylla, 766; L 92.24, as C. ochrostriata.

Ecol. \& Distr.: In eutrophic boreo-nemoral forest, August, very rare; one locality in Estonia: Jõgeva Co., Saare Comm., Roela, Vooremaa Forest Ecology Station, in spruce forest of Hepatica site type, 17 Aug 1971, K. Kalamees.

Voucher specimens studied: TAAM 081663 (det. K. Kalamees, as C. magnicapitata; rev. A. Hausknecht, as C. sienophylla ss. auct.); rev. K. Kalamees, as C. ochrostriata var. ochrostriata. Comments. In Europe almost all specimens formerly determined as $C$. sienophylla, represent C. ochrostriata.

Conocybe PILOSElla (Pers.: Fr.) Kühner, Le genre Galera: 92. 1935, var. PILOSELLA

Icon.: BK 377, as C. brunneola, 384, as C. pallidospora, 385, as C. pilosella; CD 1325, as C. 
pilosella; H 20 a-c, pp. 756-757; L 92.20.B, as C. pilosella.

Ecol. \& Distr.: In eutrophic boreo-nemoral forests, September, very rare; one locality (two records) in Estonia: Jõgeva Co., Saare Comm., Roela, Vooremaa Forest Ecology Station, in spruce forests of Hepatica site type, 23 Sep 1972, K. Kalamees.

Voucher specimens studied: TAAM 081754, 081877b (both det. A. Hausknecht, as C. pilosella); both rev. K. Kalamees, as C. pilosella var. pilosella.

Conocybe Pubescens (Gillet) Kühner, Le genre Galera: 85. 1935

Icon.: Cetto76 436, 437, as C. subovalis; H 18 a, b, pp. 737-738; L 92.44.A,B.

Ecol. \& Distr.: In mesotrophic boreal coniferous and mixed forests, especially in Oxalis forest type, in cultivated arable lands and gardens, on dung, compost or forest litter, May, June, August and September, rare.

Voucher specimens studied: TAAM 074669, 080906, 081021,141236 (det. S. Veldre, as C. pubescens), 146223.

Conocybe Pulchella (Velen.) Hauskn. \& Svrček in Hausknecht, Czech Mycol. 51: 58. 1999

C. pseudopilosella Kühner \& Watling in Watling Notes Roy. Bot. Gard. Edinburgh 38: 336. 1980. Icon.: L 92.48; Phil p. 155, as C. pseudopilosella. Ecol. \& Distr.: In coastal dune, very rare; one locality in Estonia: Lääne-Viru Co., Kunda, in seashore dune, W of Kunda harbour, on fine sand near grasses, 28 Sep 1997, J. Vauras $12894 b$.

Voucher specimens studied: TUR-A 139504.

Conocybe Rickeniana P.D. Orton, Trans. Brit. Mycol. Soc. 43: 195. 1960

Icon.: BK 387; Cetto89 2218; H 13 h, i, pp.716717; L 92.31.A-C; RH p. 436.

Ecol. \& Distr.: In eutrophic boreo-nemoral forest, October, very rare; one locality in Estonia: Jõgeva Co., Saare Comm., Roela, Vooremaa Forest Ecology Station, in spruce forest of Hepatica site type, 13 Oct 1972, K. Kalamees.

Voucher specimens studied: TAAM 081814.

Conocybe RICKEnII (Jul. Schäff.) Kühner, Le genre Galera: 115. 1935

Icon.: BK 388; Cetto89 2221, as C. crispa; Cetto93 2648; H 18 h, 22 a-c, pp. 804-806; L 92.6.B,V; Phil p. 155.
Ecol. \& Distr.: On rotting see plant debris, August, very rare; one locality in Estonia: Lääne Co., Lihula Comm., Matsalu National Park, Metsküla, on Zostera bank, 5 Aug 1979, K. Kalamees.

Voucher specimens studied: TAAM 120740 (det. K. Kalamees, as C. rickenii).

Conocybe Rostellata (Velen.) Hauskn. \& Svrček in Hausknecht, Czech Mycol. 51: 61. 1999

Icon.: H 21 b, c, pp. 760-762.

Ecol. \& Distr.: In boreo-nemoral forests, September, rare.

Voucher specimens studied: TAAM 079940, 080842; TUR-A 139439 (Hausknecht, 2005).

Conocybe SEmiglobata Kühner \& Watling, Notes Roy. Bot. Gard. Edinburgh 38: 337. 1980, var. SEMIGLOBATA

C. affinis Singer ss. Singer \& Hausknecht, Pl. Syst. Evol. 180: 87. 1992.

Icon.: BK89 390, as C. semiglobata, 394, as C. tenera; Cetto89 2216, as C. semiglobata; H 5 a-e, pp. 686-688; L 92.43.A-C, as C. semiglobata, 92.41.B, as C. tenera.

Ecol. \& Distr.: In grasslands on mineral soil and coastal grasslands, eutrophic alvar forests and juniper shrublands, wooded meadows, parks, May to September, rather rare; especially in Suur-Vilsandi Island in Saare Co.

Voucher specimens studied: H 7019145, TAAM $084548,121425,121432,121459,121464$, 121487, 121586, 122412, 141555; TUR-A 139499. All exsiccates det. A. Hausknecht, as C. semiglobata; all rev. K. Kalamees, as C. semiglobata var. semiglobata.

Conocybe subalpina (Singer) Singer $\&$ Hauskn., Pl. Syst. Evol. 180: 89. 1992

Icon.: H 1 d, 3 d, e, 20 f, pp. 677-678; L 92.42.

Ecol. \& Distr.: In eutrophic alvar mixed forests, open lawns, September, very rare; two localities in Estonia: Pärnu Co., Koonga Comm., Tammaru, in alvar oak (Quercus robur) forest with Picea abies, Betula pendula, Corylus avellana, 11 Sep 1977, L. Pihlik, M. Vaasma, K. Kalamees; Lääne-Viru Co., Sõmeru Comm., Kohala, by manor house on mowed lawn, Sep 1992, I. Lumiste.

Voucher specimens studied: TAAM 095611, 145476 .

Conocybe subovalis Kühner \& Watling, Notes Roy. Bot. Gard. Edinburgh 38: 340. 1980

Icon.: BK 393; H 2 d-f, pp. 684-686; L 92.35.A; Phil p. 155. 
Ecol. \& Distr.: In mixed forests, on coastal dunes, in grasslands, May, September and October, rare.

Voucher specimens studied: TAAM 121773; TUR-A 139481, 139486, 139488.

Conocybe subpallida Enderle, Z. Mykol. 57: 91. 1991

Icon.: Cetto93 2654; H 2 a-c, pp. 675-676; L 92.39 .

Ecol. \& Distr.: In eutrophic boreo-nemoral forests, ruderal habitats, in herb, September and October, very rare; two localities in Estonia: Jõgeva Co., Saare Comm., Roela, Vooremaa Forest Ecology Station, in spruce forest of Hepatica site type, 22 Sep 1972, K. Kalamees; Tartu Co., Tartu town, 181 Riia St., in plantation of Pseudotsuga sp., 6 Oct 1995, K. Kalamees \& M. Vaasma.

Voucher specimens studied: TAAM 113708, 142819.

Conocybe subpubescens P.D. Orton, Trans. Brit. Mycol. Soc. 43: 195. 1960

C. cryptocystis (G.F. Atk.) Singer 1954 ss. auct.;

C. digitalina (Velen.) Singer ss. auct.

Icon.: BK 379, as C. digitalina; Cetto89 2222, as C. cryptocystis; D p.582, as C. cryptocystis; H 16 a, c, pp. 730-732; L 92.46.A-D.

Ecol. \& Distr.: In eutrophic alvar forests, in cutting areas, on decaying wood, in forest litter, August, very rare; two localities in Estonia: Lääne-Viru Co., Laekvere Comm., $1.5 \mathrm{~km} \mathrm{~W}$ of Luusika, in cutting area, on wood-residues, 29 Aug 1962, H. Kelder; Saare Co., Kihelkonna Comm., Viki, in alvar mixed forest with Quercus robur, Pinus sylvestris and Juniperus communis, 12 Aug 1980, K. Kalamees.

Voucher specimens studied: TAAM 073203 (det. H. Kelder, as C. tenera), 121405.

Conocybe tenera (Schaeff.: Fr.) Fayod, Ann. Sci. Nat. (Bot.) Sér. 7, 9: 357. 1889

Icon.: H 4 a, b, pp. 682-684.

Ecol. \& Distr.: Eutrophic boreo-nemoral forest, September, very rare; one locality in Estonia: Jõgeva Co., Saare Comm., Roela, Vooremaa Forest Ecology Station, in spruce forest of Hepatica site type, 23 Sep 1972, K. Kalamees \& A. Kollom. Voucher specimens studied: TAAM 113703 (det. K. Kalamees).

Conocybe velutipes (Velen.) Hauskn. \& Svrček in Hausknecht, Czech Mycol. 51: 68. 1999 var. VELUTIPES
C. Kuehneriana Singer, Beih. Nova Hedwigia 29: 212. 1969.

Icon.: Cetto93 2657, as C. kuehneriana; H 12 b, 28 a, both as C. velutipes, pp. 779-780.

Ecol. \& Distr.: In eutrophic alvar shrubland, August, very rare; one locality in Estonia: Saare Co., Kihelkonna Comm., Vilsandi National Park, Suur-Vilsandi Island, in alvar juniper grassland, 14 Aug 1980, K. Kalamees.

Voucher specimens studied: TAAM 121430 (det. A. Hausknecht, as C. velutipes); rev. K. Kalamees, as $C$. velutipes var. velutipes.

\section{PHOLIOTINA Fayod, Ann. Sci. Nat. Sér. VII, 9: 359. 1889}

Pholiotina ARrhenil (Fr.) Singer, Sydowia Beih. 7: 77.1973

Conocybe arrhenii (Fr.) Kits van Wav., Persoonia 6: 147. 1970; Ph. blattaria ss. auct.

Icon.: BK 398; CD 1314; H 46 a-c, pp. 875-876; L 96.4.A,B,C.

Ecol. \& Distr.: In deciduous and mixed forests on mineral soils, on cultivated arable lands, along paths in grass and under shrubs, September and October, rather rare.

Voucher specimens studied: H 7019146, 7019157; TAAM 080883, 145521, 171836; TUR 122435; TUR-A 139383, 183486, 195575 (all det. A. Hausknecht, J. Vauras or I. Kytövuori, as $P h$. arrhenit).

Pholiotina BRunnea (Watling) Singer, Beih. Sydowia 7: 79. 1973

Conocybe brunnea Watling, Persoonia 6: 318. 1971.

Icon.: CD 1318; Cetto89 2229; H 42 e, h, pp. 862-864; L 96.13.A,B.

Ecol. \& Distr.: In eutrophic boreo-nemoral forests, in herbal layer, on leaf litter or rotten wood, September, very rare; two localities in Estonia: Saare Co., Kaarma Comm., Abruka Nature Reserve, Abruka Island, in broad-leaved forest of Aegopodium site type, 14 Sep 1993, J. Vauras 8821 (Hausknecht 2009: 609); Saare Co., Torgu Comm., Viieristi Nature Reserve, W of Koltsi Lake, deciduous forest with Quercus robur, Corylus avellana, Betula, Picea abies and Ulmus glabra, on calcareous mull soil, 20 Sep 2008, J. Vauras 26606.

Voucher specimens studied: TUR 110454 (det. J. Vauras, as Conocybe brunnea); TUR-A 182356 (det. J. Vauras, as Ph. brunnea). 
Pholiotina Coprophila (Kühner) Singer, Trud. Bot. Inst. Komarowa Akad. Nauk SSSR II/6: 434. 1951

Conocybe coprophila (Kühner) Kühner, Le genre Galera: 125. 1935.

Icon.: BK 400; H 39 e, f, pp. 849-850; L 96.9.

Ecol. \& Distr.: In pasture, on dung, August, very rare; one locality in Estonia: Lääne Co., Nõva Comm., Rannaküla, in pasture by Alnus forest, on Bos taurus dung, 20 Aug 1968, K. Kalamees (Hausknecht, 2009: 563).

Voucher specimens studied: TAAM 077501 (det. K. Kalamees).

Pholiotina Dasypus (Romagn.) P.-A. Moreau, Fungal Diversity 20: 135. 2005

Ph. utriformis (P.D. Orton) Bon, Doc. Mycol. 21/83: 39. 1991; Ph. subnuda (Kühner \& Watling) Bon, Doc. Mycol. 21/38: 39. 1991; Ph. subverrucispora (J. Veselský \& Watling) M.M. Moser, Kleine Kryptogamenflora IIb/2, 4. ed.: 283. 1978.

Icon.: H 42 a-d, pp. 860-861; L 96.12.A,B,C, as Ph. utriformis.

Ecol. \& Distr.: October, very rare; one locality in Estonia: Võrumaa Co., Haanja Comm., Uigumägi, sloping grass-herb spruce forest with Betula, Populus tremula, Corylus, Salix etc., 1 Oct 1997, I. Kytövuori 97-2092.

Voucher specimens studied: H 7019156 (det. I. Kytövuori, as Conocybe subverrucispora, conf. J. Vauras as Ph. dasypus).

Pholiotina filipes (G.F. Atk.) Singer, Trud. Bot. Inst. Komarowa Akad. Nauk SSSR II/6: 435. 1951

Ph. aberrans (Kühner) Singer, Trud. Bot. Inst. Komarowa Akad. Nauk SSSR II/6: 436. 1951; Ph. sulcatipes (Peck) Bon ss. Hausknecht in Horak, Röhrlinge und Blätterpilze in Europa: 321. 2005.

Icon.: Cetto89 2228, as Ph. sulcatipes; H 38 g, h, pp. 854-855; L 96.17.A,B, as Ph. aberrans

Ecol. \& Distr.: In eutrophic boreo-nemoral forest, July, October, very rare; one locality (two records) in Estonia: Jõgeva Co., Saare Comm., Roela, Vooremaa Forest Ecology Station, in spruce forest of Hepatica site type, 10 July 1972, K. Kalamees, 3 Oct. 1972, K. Kalamees \& A. Kollom (Hausknecht, 2009: 580).

Voucher specimens studied: TAAM 081981 (det. A. Hausknecht, as Ph. sulcatipes), 114232 (det. A. Hausknecht, as Ph. sulcatipes); rev. K. Kalamees, as Ph. filipes.
Pholiotina mairei (Watling) Enderle, Beitr. Kenntn. Pilze Mitteleur. 2: 113. 1986. var. MAIREI Icon.: Cetto93 2665, as Ph. mairei; H 41 a, b, pp. 856-857, as Ph. mairei; L 96. 15. B, as Ph. mairei.

Ecol. \& Distr.: in orchards, June, very rare; one locality in Estonia: Tartu Co., Tartu town, 9 Palsami St., in orchard, on humid soil, 5 June 1996, K. Kalamees.

Voucher specimens studied: TAAM 146905 (det. A. Hausknecht, as Ph. mairei); rev. K. Kalamees, as $P h$. mairei var. mairei.

Pholiotina nemoralis (Harmaja) Bon, Docums Mycol. 21/83: 38. 1991, var. DENTATOMARGINATA (Watling) Hauskn., Österr. Z. Pilzk. 16:92. 2007 $P h$. dentatomarginata (Watling) Enderle, Beitr. Kenntn. Pilze Mitteleur. 2: 142. 1986.

Conocybe appendiculata Kühner forma macrospore, Le genre Galera:149. 1935, nom. nud. Icon.: D p.584, as Conocybe appendiculata; $\mathrm{H}$ $43 \mathrm{~g}, \mathrm{~h}, \mathrm{pp} .871-872$.

Ecol. \& Distr.: in eutrophic Filipendula paludifying deciduous forests and in ruderal habitats (land improvement areas on moist soil among grasses and Carex), May, rare.

Voucher specimens studied: TAAM 080017b, 084545 (Hausknecht, 2009), 141147, 141154; all det. A. Hausknecht, as Ph. nemoralis; all rev. K. Kalamees, as $P h$. nemoralis var. dentatomarginata.

Pholiotina teneroides (J.E. Lange) Singer, Beih. Bot. Zbl. 56B: 170. 1936

Conocybe teneroides (J.E. Lange) Kits van Wav., Persoonia 6: 160. 1970

Pholiotina blattaria (Fr.) Fayod, Annls Sci. Nat., Bot. ser. 7(9): 359. 1889

Pholiotina percincta (P.D. Orton) Bon, Docums Mycol. 21/83: 38. 1991.

Icon.: BK 403; Cetto89 2231; D p. 583; H 46 g, h, pp. 884-885; L 96.3.A, B; Phil p. 156, as C. percincta.

Ecol. \& Distr.: In ruderal habitat, September, very rare, one locality in Estonia: Hiiu Co., PihlaKaibaldi Nature Reserve, Mäeselja, on garage earth floor, 16 Sep 2001, S. Huhtinen 01/57 (det. S. Huhtinen, as Pholiotina blattaria, det. J. Vauras, as Ph. teneroides 2012).

Voucher specimens studied: TUR 157816.

Comments: Pholiotina teneroides is the only species in the genus Pholiotina with basidia with 2 sterigmata. 
Pholiotina velata (Velen.) Hauskn., Czech Mycol. 51: 66. 1999

Ph. appendiculata (Watling) Singer, Beih. Sydowia 7: 79. 1973; Conocybe appendiculata Watling, Persoonia 6:329. 1971.

Icon.: BK 397, as C. appendiculata; Cetto89 2232, as Ph. appendiculata; CD 1317, as $\mathrm{Ph}$. appendiculata; H 43 a, b, pp. 866868; L 96.18.B, as Pholiotina nemoralis. Ecol. \& Distr.: In eutrophic boreo-nemoral forest, September, very rare; one locality in Estonia: Saare Co., Kaarma Comm., Abruka Nature Reserve, Abruka Island, in broad-leaved forest of Aegopodium site type, 14 Sep 1993, J. Vauras 8838 (det. J. Vauras, as Conocybe brunnea, det. A. Hausknecht, as Ph. velata).

Voucher specimens studied: TUR 110432.

Pholiotina vestita (Fr.) Singer, Beih. Bot. Zentralbl. 56B: 170. 1936

Conocybe vestita (Fr.) Kühner, Le genre Galera: 155. 1935.

Icon.: BK 404, as Conocybe vestita; C93 2664; CD 1316; H. 42 i-k, pp. 864-866; L 96.14.A,B. Ecol. \& Distr.: In eutrophic boreo-nemoral forest, September, very rare; one locality in Estonia: Hiiu Co., Pühalepa Comm., Hiiumaa Islets Landscape Reserve, W part of Hanikatsi Island, in deciduous forest with Betula, Quercus robur, Tilia cordata and abundant Juniperus communis, under Tilia cordata on calcareous soil, 24 Sep 2008, J. Vauras 26681.

Voucher specimens studied: TUR-A 182482 (det. J. Vauras, as Ph. vestita).

\section{REFERENCES}

Breitenbach, J. \& Kränzlin, F. 1995. Pilze der Schweiz 4. Blätterpilze. 2. Teil. Luzern.

Cetto, B. 1976. I Funghi dal vero 2. Trento.

Cetto, B. 1987. I Funghi dal vero 5. Trento.

Cetto, B. 1989. I Funghi dal vero 6. Trento.

Cetto, B. 1993. I Funghi dal vero 7. Trento.

Courtecuisse, R. \& Duhem, B. 1994, 2000. Guide des champignons de France et d'Europe. Lausanne-Paris.

Dähncke, R. M. 2001-2004. 1200 Pilze in Farbfotos. Bechtermünz.

Hausknecht, A. 2005. Beiträge zur Kenntnis der Bolbitiaceae 10. Conocybe Sektion Pilosellae. Österr. Z. Pilzk. 14: 191-274.

Hausknecht, A. 2009. A monograph of the genera Conocybe Fayod, Pholiotina Fayod in Europe. Alassio.

Hausknecht, A., Vauras, J., Kytövuori, I. \& Ohenoja, E. 2005. Die Gattung Conocybe in Finnland. Karstenia 45: 1-32.

Hausknecht, A., Weholt, Ø., Bendiksen, E. \& KrisaiGreihuber, I. 2011. The genera Bolbitius, Conocybe and Pholiotina (Bolbitiaceae, Agaricales) in Norway. Agarica 31: 89-122.

Hausknecht, A. \& Vesterholt, J. 2008a. Conocybe Fayod. In Knudsen, H. \& Vesterholt, J. (Eds). Funga Nordica. Agaricoid, boletoid and cyphelloid genera. Pp. 626-645. Copenhagen.

Hausknecht, A. \& Vesterholt, J. 2008b. Pholiotina Fayod. In Knudsen, H. \& Vesterholt, J. (Eds). Funga Nordica. Agaricoid, boletoid and cyphelloid genera. Pp. 651-657. Copenhagen.

Ludwig, E. 2007. Pilzkompendium. Band 2. Abbildungen. Berlin.

Paal, J. 1997. Classification of Estonian vegetation site types. Tallinn.

Phillips, R. 1981. Mushrooms and other fungi of Great Britain and Europe. London.

Ryman, S. \& Holmåsen, I. 1984. Svampar: en fälthandbok. Stockholm. 
40 Folia Cryptog. Estonica 